\title{
Experiences of Family Caregivers' Involvement in Treatment Related- Decision-Making in Triadic Health Encounters
}

\author{
Enaam Al-Ananbeh ${ }^{1}$, Abeer Al-Wahadneh ${ }^{2}$ \\ ${ }^{1}$ PhD. ANS. RN, Assistant Dean of Princess Muna College of Nursing, Mutaa University, Amman, \\ Jordan. \\ ${ }^{2}$ MSN. RN, Princess Muna College of Nursing, Mutaa University, Amman, Jordan
}

\section{Article Info \\ Article history: \\ Received:08 September 2020 \\ Revised: 28 October 2020 \\ Accepted: 28 October 2020 \\ Keywords: \\ Family Roles, \\ Chronic Diseases, \\ Family Caregiver. \\ Paper Type : \\ Research Article \\ Corresponding Author: \\ Enaam Al-Ananbeh \\ Email: \\ Enaam enab@yahoo.com}

\begin{abstract}
Purpose: To explore the experiences of family caregiver in health decision-making for patients with chronic diseases.

Study Design: Qualitative Descriptive Design.

Subjects and Methods: A descriptive qualitative approach was used. A purposive sample of fifteen family caregiver for patients with chronic diseases were interviewed in Amman, Jordan; fifteen caregivers; males $(n=2)$, females $(n=13)$. Average of age $=40$ years old. Data were generated through phone messages voice records over a period of two months (March \& April 2020) in Amman, Jordan. Data were analyzed using a five-step technique proposed by Giorgi (1985).

Results: The findings of the study revealed that three major themes related to family caregivers' experiences in health decision-making for patients with chronic diseases: 1) The patient has the right to decide about his health, 2) Healthcare providers know better, and 3) Roles of family caregivers in the decision making process.

Conclusion: The vital role of the family members in taking decisions for patients with chronic diseases is well-recognized by healthcare providers. Continuous systematic assessment of family members' preferences and needs is crucial to provide the needed support for their patients in decision-making
\end{abstract}

\section{Introduction}

In the last decade, the role of family caregivers started to be highlighted, in response to the limits of shared decision-making models focusing only on patient-physician interactions (Kristopher, 2017). Some patients are unsure of the value of their own decision and wanted to leave the decision to others (Olssonet al., 2016). Recently, discharge rates of hospitalized patients are increasingly high. Therefore, elderly with chronic diseases are living longer. Many patients with chronic diseases live with their families who care for them. Family members who provide care for their patients at home are called family caregivers (FCs). A family caregiver is any relative, partner, friend or neighbour who has a significant personal relationship with, and provides a broad range of assistance for an older person or an adult with a chronic or disabling condition. These individuals may be primary or secondary caregivers and live with, or separately from, the person receiving care(Alliance, 2015).

Patient and family centred-care and shared decision making approaches reflect and accelerate the shifting roles of patients and families in health care as they become more active, informed, and influential(Carman et al., 2013; Chong et al., 2015). Many international 
health laws call for patients' rights that ensure patient autonomy (Lamoreet al., 2017; Doumit et al., 2008; Hirpara et al., 2016) ). Excluding competent patients from the involvement in treatment decision-making (D.M) process violates the Code of Ethics of the Singapore Medical Council as patient autonomy and confidentiality were considered breached(Chong et al., 2015).

Involvement in treatment health D.M is influenced by cultural differences of patients and health care providers (HCPs) (Chong et al., 2015; Lin et al., 2013).In the Asian culture, which entails the need for engaging and interacting in a network of relations with others, as opposed to the western definition whereby decisions are solely the prerogative of the individual (Chong et al., 2015). Patient autonomy is not considered an absolute in some countries as Japan, Korea or China where treatment decision making is usually the domain of the family members and clinician (Chong et al., 2015). Similarly, in the Middle East, the patients' autonomy was well recognized by the physicians. The patients' involvement in decision making was affected by many factors include: religious, gender, and educational level. But the patients still preferred the shared family decision making model. (AlHaqwi et al., 2015; Hammami et al., 2020)

Family caregivers' behaviours during D.M process may be seen as a continuum from a passive to an active attitude. Most of the patients, FCs and physicians prefer the FCs to be involved in treatment decision-making(Dionne-Odom et al., 2019; Lamore et al., 2017). Patients' decisions are influenced by the judgment of significant others as parents, spouse and friends(Fishbein, 1975; Icek, 1991; Javadi et al., 2013). Patients may discuss important considerations with their physician and family. Then the patient, family, and physician would work together to develop and initiate a treatment approach that take in consideration both the patient's and family's emotional status about the disease, treatment preferences and goals, life circumstances, values, and risk tolerance for adverse outcomes Family members as caregivers often play different roles in caring for their patients (Chong et al., 2015; Doumit et al., 2008; Gainer et al., 2017; Lamore et al., 2017; Laryionava et al., 2018; Quinn et al., 2015). Lamore, (2017) identified four subthemes regarding roles of the family during chronic diseases, including role of social support, role of intermediary, role of collaborator and role of messenger.

Tradition dictates that family members accompany the patient to the hospital and make frequent visits to the hospital and later at home. When a person is seriously ill, a family member stays with the patient constantly (Doumit et al., 2008). Family caregivers may influence treatment-related decisions for their patients through direct or indirect ways. Acting as mediaries between the patient and HCPs is an example of direct influence (Lamore et al., 2017).Role of mediator includes communication, asking questions and providing information. Accurate information help reduce uncertainty and empower caregivers by giving them a sense of control (Supportive, 2019). Whereas, indirect influence denotes many aspects, one might include just the presence of family caregivers during health encounters (Lamore et al., 2017). A qualitative study with 37 patients with advanced cancer and 40 caregivers conducted by Zhang et al. (2005) showed that $65 \%$ of families had disagreements regarding treatment decisions including discontinuation of anti-cancer treatment, thus considerably influencing patients' treatment choices (Laryionava et al., 2018).

Copyright (C) 2020, Journal of Scientific Research in Medical and Biological Sciences (JSRMBS), Under 
FCs often play key roles with patients during their health encounters and are involved in discussions regarding treatment plans (Lamore et al., 2017). They are often present and very active in treatment decision-making encounters, providing assistance, informational and emotional social support(Dionne-Odom et al., 2019).

The treatment decision-making process can include different specific roles of FCs such as: (1) gathering and interpreting information; (2) motivating the patient to ask HCPs certain questions; (3) advising the patient which treatment option to select; (4) advocating on the patient's behalf regarding the timing or place of treatment or the patient priority in receiving treatment; (5) supporting the patient's treatment decision. The involvement of family members in treatment decision-making may be particularly important for patients with chronic diseases because of the stress related to the diagnosis, the uncertain outcome, and the potentially major impact of the illness trajectory and treatment management on other family as a whole (Charles et al., 1997).

Patient- and family-centred care represents the future model of healthcare. This approach focuses on the involvement of the entire family in the D.M process (McCabeBennett et al., 2014). The triad relationship of patient, HCPs and FC and discussion of treatment options for the long-term management of a chronic illness is different from the situation of an acute case.

In acute health status, the patient needs to make an important treatment decision under limited time period. In chronic diseases, multiple treatment options may be available (e.g., medications options, surgical interventions, transplantation) consequently, treatment-related decisions should be made. Worldwide, limited studies of FCs involvement in treatment decision making were conducted. The literature reveals studies regarding specific chronic diseases mainly cancer and kidney problems. However, little is known about FCs involvement in treatment D.M of other chronic diseases. Up to the authors' knowledge, no studies were done in Jordan in this regard. Therefore, this study aims to explore FCs experience of their involvement in treatment-related D.M during health encounters.

Shared decision-making models were limited to focus only on patient-physician interactions. Family involvement is a more recent development compared to dyadic bilateral (patient-HCPs) approaches in which the FCs' role is rarely addressed. Limited research studies have been conducted on FCs experiences in involvement in treatment-related decisions. Hence, the purpose of this study is to explore the experiences of FCs involvement in treatment-related decisions for adult patients with different chronic diseases. The guiding question in the study is: How do FCs describe their experiences regarding their involvement in treatment-related D.M for patients with chronic diseases?

\section{Methodology and Procedures}

\section{Design}

The present study used a descriptive qualitative design to describe the phenomena under study. The methodology of Georgi (1985) was adopted for the analysis process as well as steps suggested by Guba and Lincolin 1985 were utilized to address rigor of study methods. 


\section{Sampling}

A purposeful sampling of Family Caregivers caring for patients with chronic diseases was interviewed. The recent conditions regarding global Corona virus-COVID-19 have changed the authors' plan of study method. To ensure physical distance with the family caregivers, the contact number of the primary researcher was reviewed, and a phone message was sent to FCs by a social media application.

The messages contain the invitation statement and interview guides. The inclusion criteria of participating Family Caregivers include those who are adults and care of or live with a patient complaining of chronic diseases. Written informed consent for participants was replaced by agreement on the invitation statement that was typed as a short phone message on a specific social media application. The statement was individually sent to all 15 participants.

\section{Sample Description}

The sample consisted of 13 females and 2 males. Their age range was 25 to 56 years, with a mean age of 40 years. Participants' lived experience with the role of caregiver for a chronic patient ranged between 1 and 8 years. Regarding FCs Relationship with their patients; Spouse $=6$ and parental $=9$.

\section{Interviews}

All phone interviews were conducted privately by the primary researcher.The interviews were audio taped and transcribed verbatim. Increasing the sample numbers is limited by the contact numbers availability with the researcher. Further, the sample is a purposeful one and it's the most suitable sampling technique for qualitative researches. Such types of samples are always small consistent with the purpose of the recent study.

\section{Data Generation}

Data was generated through semi-structured, mobile phone interviews using an interview guide which was confirmed by a panel of experts in qualitative approaches. Data generation was conducted over a period of two months between March and April, 2020. This approach allows the interviewer to provide a broad question and then guide participants through probes to describe their involvement experience. All fifteen family caregivers were interviewed in Arabic by the primary researcher. Prior to initiating the study, the researcher tried out questions from the interview guide with individuals having similar characteristics to those included in the study to make sure that they were understandable.

Each interview as voice record was heard and transcribed verbatim into a separate Word document in Arabic. After each interview, demographical data was obtained for both family caregivers and their patients (Table 1). Transcripts of interviews and analysis files were kept in a double locked cabinet at Mutah University, Jordan. Pseudonyms were used for each participant in the study report to ensure confidentiality. 
Table 1: Demographic Characteristics of the Participants

\begin{tabular}{|c|c|c|}
\hline A-Demographical details of the participating family caregivers & $\mathrm{N}(15)$ & ) \\
\hline \multicolumn{3}{|l|}{ 1-Age (Years) } \\
\hline $25-35$ & 3 & 20 \\
\hline $36-46$ & 5 & 33.3 \\
\hline $47-57$ & 7 & 46.6 \\
\hline \multicolumn{3}{|l|}{ 2-Gender } \\
\hline Male & 2 & 13.33 \\
\hline Female & 13 & 86.6 \\
\hline \multicolumn{3}{|l|}{ 3-Relationship with patient } \\
\hline Spouse & 6 & 40 \\
\hline Parental & 9 & 60 \\
\hline \multicolumn{3}{|l|}{ 4-Marital status } \\
\hline Married & 12 & 80 \\
\hline Single & 2 & 13.33 \\
\hline Divorced & 1 & 6.66 \\
\hline Others & . & \\
\hline \multicolumn{3}{|l|}{ 5-Level of education } \\
\hline School & 4 & 26.66 \\
\hline Diploma & 6 & 40 \\
\hline University. & 5 & 33.3 \\
\hline \multicolumn{3}{|l|}{ 6-Health status } \\
\hline No disease & 10 & 66.66 \\
\hline DM & 1 & 6.66 \\
\hline HTN & 1 & 6.66 \\
\hline Others & 3 & 20 \\
\hline
\end{tabular}


7-Employment

Employed

7

46.6

Not Employment

9

60

8-Participants' years of experiences as caregiver

All above 5 years of experience

B-Demographical details of the participating Patients :

1-Age (Years)

$40-50$

2

$51-61$

5

33.3

$62-72$

7

46.6

$73-83$

1

6.66

2-Gender

11

73.2

Male

4

26.66

Female

3-Level of education

Illiterate

1

6.66

School

10

66.66

Diploma

3

20

University.

1

6.66

4-Health status(chronic diseases)

Cancer

1

6.66

Non cancer

14

93.6

5-Living conditions

With family

12

80

With spouse

3

20

Alone

None

Copyright (C) 2020, Journal of Scientific Research in Medical and Biological Sciences (JSRMBS), Under 
6-Insurance type

Military

Civilian

1

6.66

Others

none

Total

15

100

Source: Authors

Each interview was coded so that only the researcher had knowledge of who the participants were and what they contributed. Participants were assured that they could end the interview at any time. None of the participants ended an interview session due to any cause.

\section{Ethical issues}

Approval to conduct the study was obtained from the institutional review board at the RMS and Mutah University. Each participant was assured that his/her responses would be confidential, and his/her participation was on a voluntary basis. Written informed consent for participants was replaced by agreement on the invitation statement that was typed as a short phone message on a specific social media application. The statement was individually sent to all 15 participants.

\section{Rigor}

Steps suggested by Guba and Lincoln (1985) were taken to address rigor of study methods. Credibility was achieved by within-method triangulation for data collection, peer debriefing, and member checks. Transferability was assured by the use of thick description from the participants' interviews. Dependability and conformability were secured by doing intercoder reliability, as suggested by Polit and Beck (Polit \& Beck, 2012).

The researcher (coder) induced themes through qualitative approach. The identified themes were then compared with those of a second researcher for understanding. The agreement between the two researchers was around 95\%; the remaining themes were reviewed, and remaining disagreements were resolved.

\section{Data Analysis}

Data was analyzed using a five-step technique proposed by Giorgi (1985) to identify themes related to the phenomena under study. Each recorded voice message was repeatedly heard and reviewed. The researcher then read and read each transcribed message to get a sense of the whole experience, then divided data into manageable parts that reflect the participants own meaning of the experience. Three experts in qualitative approach checked the initial list of codes and concluded an agreement upon set of codes. The coded transcripts were then reviewed by two other experts for consistency, and disagreement was resolved through group discussion. 
The researcher identified quotes that best illustrated common themes and included them in the results of this study. Quotes were translated into English by bilingual translators and were edited to ease reading.

\section{Results and Discussion}

Three core themes were identified. These themes are not presented in order of importance.

\section{The Patient has the Right to Decide About His Health}

More recently, respect for patient autonomy, as articulated by the Institute of Medicine in its call for "Patient-Centred Care", has stimulated ways to optimally provide information about risks, benefits and treatment options and facilitate patient control over decision making about their care (Gainer et al., 2017). FC behaviours during D.M process may be seen as a continuum from a passive to an active attitude. A passive attitude illustrates that FCs are not involved in the D.M process for some reasons. One reason was that the family caregivers believe in the principle of patients' right to make his/her own decision, as the final decision should be left to the patient himself.

One participant (F4), a 37 years old wife who provides care for her husband reported that "I observe my husband and accompany him to visit his physician. My role is more as observer rather than as a participant in the treatment plan or health decision. The final decision is left to him since this decision is closely related to the patient not to the person who accompany him". Another participant (F11), a FC who cares for her husband expressed that all decisions are left for him deference to his preferences. As sometimes he takes unhealthy decisions and refuses to change them. F11 said " when I give him any advice to do or avoid something, he gets nervous and tells me that it's not of my business.... I do my best to manage his anger and nervousness so I could not involve directly in his decisions." she added: he is number one (in the family) and he is the one who decide..).

These findings implies FC passive role in the final decisions as it's the patients' right to be self-determined regarding their final decision. Whereas, FC usually participates (directly or indirectly) in the process of D.M including asking questions, support, and helping their patients to formulate their final decision. On the other hand, if the patient is passive during the D.M process, then FCs play a more active role. (Lamore et al., 2017)

\section{Health Care Providers Know Better}

Patients as well as FCs could leave the decision to HCPs when they trust them. Some patients underestimate their family member's role in making or even sharing the decision. They express that their FCs lack health knowledge compared to HCP's knowledge that authorizes them to take the decision (Al-ananbeh et al., 2018).

Some lack the health knowledge and do not understand HCPs medical terms therefore they do not participate or discuss their patients' health status with HCPs. As reported by (F8) who was caring for her mother "I do not ask her Doctor as I do not know what to ask, they speak medical and English terms." Further, a RN (F9) said that "I did not have the sufficient knowledge to discuss my parents health status with their Doctors, I was newly graduated 
$R N . "$ Another female (F7) experienced feelings of embarrassment to ask about her patient health and she said: "They (i.e. HCPs) talk with each other in English, I feel embarrassed to ask them."

Further, findings of this study revealed that some FCs expressed their full trust in HCPs knowledge and their ability to make the final decision. (F13) said that "It's their (i.e. physicians) job they know more (than us as FCs).I do not want to interfere with their job." Based on HCPs specialized knowledge they provide different health options to patients and their families. FCs trusted HCPs to let them choose the best option for their patients' welfare.

\section{Roles of FCs in Decision-Making Process.}

\section{Advocator Role}

FCs can give the needed information to HCPs and ask questions to best understand their patients' health conditions. FC who had active attitudes and who play an intermediary role between their patients and physicians are usually involved in dyadic D.M process with HCPs. Two female nurses (F1, F10) said "I tell the physician about her (mother) condition... and this save his (Dr) time and effort and help him to determine what the best is for the patient."

\section{Informational Support}

The patients' family is often a great source of information. Developing a trusted therapeutic relationship between HCPs and the patients' family is important for patient care (McCabe-Bennett et al., 2014). This engagement meant a lot because it built a foundation of trust and friendship, which was a comforting sense of familiarity (Boyle, 2015). Participant in this study shared their thoughts, ideas, and participate in discussion. They tend to summarize health information given by HCPs to their patients and repeat or filter it.

Some FCs $(F 1,3,5,7,8,9,10,11$, and 12) expressed their active involvement in the D.M process by discussing and asking questions. F3 said:"We ask him (the Dr) why you should admit him (the patient), we ask about everything, about medication: what are these drugs and why... why he (the patient) needs a major surgery?"

In major decisions such as surgical operation, FC and patient might be unable to formulate the final decision; rather they seek other resources as trusted HCPS. Conflict may come out during their discussion, but a final decision should be taken. Some FCs (6 participants) sought external resources to understand the case and to be able to make the final decision. F3 stated that "When he decided to amputate my father's leg, I told him I will not give you an instant answer but I should consult my sisters and brothers, who in their turn will ask another physician and seek information through the internet to get more understanding about the operation."

Physician-FC relationship influences the patients' decision. They may discuss the treatment choices excluding the patient. FC may provide brief information to the patient and then filter them if were inconsistent with the patients' preference, so as not to distress him/her.

Copyright (C) 2020, Journal of Scientific Research in Medical and Biological Sciences (JSRMBS), Under 
F5 stated that "... it was a risky operation, thus I discussed it with the Dr to find an option with the least risk on him, he (Dr.) agreed with my opinion (to avoid the operation) even that decision was against my husband preferences and finally, we convinced him."

Communication is vital when developing a therapeutic relationship between HCPs and the patients' family (McCabe-Bennett et al., 2014). FCs need information during the D.M process in order to help and support their patient. They need information regarding treatment, outcome of treatment, impact of treatment on family and patient lifestyle as well as financial issues. The information given should be understandable and within patients and FCs preferences.

F7 declared that "I could not understand anything as he talked in English and I do not ask him what he was talking about as I feel shy (I don't understand medical terms) and I had a bad experience in this regard. Once, when they were discussing her condition in English, I asked him whats up with my mother, is there something serious? He replied me: this is between us the doctors." F8 said that: "She was afraid to insert the arterio-venous (AV) shunt and refused it, she was tired and in need to start renal dialysis, we encouraged her and the Dr. frightened her if she insisted to avoid the shunt her health might get worsen. We coalited with the Dr to convince her and she finally agreed."

Most of the participants expressed their willingness to provide social support for their patients. They usually accompany them to health care settings, follow up health appointments and treatments, spend time and efforts to care about them, and ease their living conditions. Which considered indirect involvement of FC in D.M. It doesn't embrace specific behavior, rather their voice and presence in the health setting despite their poor healthcare background (Boyle, 2015).

For instance, the presence of a FC during the health encounter may influence the patient decision. Some FCs (spouse) avoid talking with HCPs about special topics in front of their patients considering their emotions and feelings. F12, F5, and F11 provided emotional support by different ways. A 43 years old wife (F12) stated that: "I discussed our spousal relationship without his presence (her husband) to avoid any embarrassment and feeling of weakness...."

Another type of support is a financial support. Some FC (F3, 5, 7, 8, and 11) provide financial support for their patients. For instant, $a R N$ (F5) quit her job to stay beside her ill husband. She said: "My husband is very tired now, he's totally dependent on me as I provide him with basic daily needs, I compiled to leave my job and use my pension to live and complete our life. We share together any decision." Another participant is a governmental employee living with her husband and their two children. Her husband is unemployed and totally dependent on his wife. She said: "I support my family by a modest salary and bear the burdens to keep the unity of my family. I accompany him, provide all of his needs as medication, follow-up... furthermore all the decisions are limited to him."

Most Family members provide social, financial, physiologic and psychological support. One participant, a 50 years old divorced female (F7) living with her elderly mother, expressed her full support (social, emotional, physical and financial support) to her mother. They are covered with military health insurance. She said: "we provide her all needs, 
sometimes she refuses to take the medication of different colors compared with the usual ones, so we buy the medication that she got used to take to please her."

\section{Discussion}

The current study presented FCs experiences in health D.M process. The emerging themes of this study are consistent with the results of previous studies (AlHaqwi et al., 2015; Chong et al., 2015; Gainer et al., 2017),but provide more in depth understanding of this phenomena. A variety of experiences were addressed giving consideration to the factors that influenced these experiences. Two major themes that were emerged in the present study were extensively described in the literature; HCPs know better and roles of FCs in D.M process.

\section{Patient Has the Right to Decide About His Health}

Both legally and ethically, Western culture favours an individual patients' right to self-determination over the beneficent protection offered by others (Lin et al., 2013).Adults are believed to be competent to make their own decisions even though someone else might be a better decision-maker for them (Barstow et al., 2018). Leaving the decision to patients themselves as it is one of their rights is well documented in the literature. (Al-Bahri et al., 2017; AlHaqwi et al., 2015; Banning et al., 2009; Chong et al., 2015; Gainer et al., 2017; Lamore et al., 2017; Sekimoto et al., 2004)

In Asian societies, the healthcare decision-making process tends to prioritize the family above the individual (Al-Bahri et al., 2017; Chong et al., 2015).The Asian cultural perception and attitudes towards illness and death mean that a family centric rather than patient centric approach is employed (Chong et al., 2015). Jordanian caregivers' desire to support their patient may explain why families are usually involved during decision making. Similar experiences of relational autonomy including engagingand interacting in a network of relations with others have been reported by others(Al-Bahri et al., 2017; AlHaqwi et al., 2015; Chong et al., 2015; Laryionava et al., 2018; Quinn et al., 2015).

Most of the participating FCs in this study experienced active roles in involvement during the D.M process in spite of recognizing their patient's right to decide. Such experience was addressed by Chong et al, (2015), who reported that patient autonomy is not considered an absolute in Japan, Korea or China where treatment D.M is usually the domain of the family members and HCPs. Leaving all D.M to the family and HCPs, is an acceptable and prevalent practice, they added. Consistency in findings emphasized the importance of patientand family- centered care and FCs involvement in health D.M which were evident in the findings of this study.

The type and nature of Family Caregiver-patient relationship could affect the degree of Family Caregiver involvement in the decision-making. Good FC-Patient relationship contributes to active and supportive involvement of FC in D.M process as was clarified in most of the participants' experiences. However, a 46 years old wife (F11) experienced a total surrender to her husband preferences regarding health decisions. She complained of poor spousal relationship with her husband who complains of different chronic diseases. She was unable to participate in any decision but left all health decisions to him as he would get angry 
and nervous which in turn negatively affects their family life. Eventually, the unstable spousal relationships lead the wife to refrain from any direct involvement in the health decisions to avoid any conflict between them (her and her husband). Such experience echo the dominant role of males in eastern countries compared with western ones. Jordan as one of the eastern countries represents such dominant role. These findings are consistent with that of Al-Bahriet al., (2017) who reported that in some Arabic countries, male family members held the dominant role in the D.M process.

In eastern cultures, sick individuals are overprotected by family members, who are always surrounding the patients (Doumit et al., 2008). In agreement with this study findings, Dionne-Odom (2019) reported that family caregivers who are not involved in helping patients make decisions may heighten the patient's distress and lead to the receipt of care and treatments inconsistent with the patient's values and preferences of their patients (DionneOdom et al., 2019).

Consequently, factors such as culture, gender, type and nature of patient-FC relationship are factors affecting the level of FC involvement in triadic-health decisions. In major decisions when the competent patient is unable to be self-determined, FC may act different behaviours. Some participants in this study sought external resources, shared the decision options with all family members and significant others. Upon agreement on a specific decision, FC then persuades the patient about the final decision.

These findings were supported with previous studies (Al-Bahri et al., 2017; AlHaqwi et al., 2015; Chong et al., 2015), who concluded the vital role of FC in involvement in D.M process in spite of patients right to decide. Such results could be explained by the fact that patients in Eastern countries are more likely directed by their families during the D.M process.

FC (F12) experiences a major role in D.M process during her husband's critical condition. Findings of this study further confirmed the findings of Chong et al., (2015); Laryionava et al, (2018) in which they found out that patients became less involved in their healthcare decision-making as their disease progressed. Consistency in findings emphasized the importance of patient- family centred care in involvement in D.M process which was evident in the findings of this study.

\section{HCPs Know Better}

The traditional paternalistic model of medical practice is still dominant in many healthcare sectors in Jordan, especially the public sector, which might have played a role in the patients' and FC preferences. Jordanians still view physicians and other HCPs as having the ultimate control over specialized health knowledge.(R. F. Obeidat et al., 2013)

A participant (F15) reported that it's the HCPs role neither hers nor any family member's. They cannot decide or even discuss any health issue. She expressed that HCPs know better, than non HCPs in their specialty, and therefore they can determine the best decision. On the other hand, most of FCs with specialized health backgrounds often play an active role in triadic health encounters (Al-Bahri et al., 2017). For instance, a retired registered nurse (F10), who have had specialized health knowledge, expressed her ability to

Copyright (C) 2020, Journal of Scientific Research in Medical and Biological Sciences (JSRMBS), Under 
play an active mediator and facilitator roles during the triadic health encounter. She discussed with her mother's Dr., and convinced him to change the treatment plan that he set before.

Surprisingly, some FCs expressed their passive role in the D.M process and reported that they do not discuss or even ask HCPs about their patients' health condition. Such passive role is related to different causes that included poor health knowledge and medical term use as reported byF8, F9, as well as F7 who experienced feelings of embarrassment to ask about her patient health. One explanation to such passive roles is the patients' and their families' perception that their relationship with their HCPs would be at risk if they disagree with their choices. Therefore, they refuse to voice this disagreement (Teh et al., 2009).

Similarly, one participant (specialized RN) in this study expressed her cautiousness to challenge physicians' authority. She preferred to be involved indirectly in making decision by offering suggestions and asking questions only. As she experienced a feeling of misunderstanding from HCPs who might consider her involvement in health decisions an interference with their job as she reported. Additionally, Al-Bahri et al, (2017) found that in spite of many physicians accepting family involvement in D.M process, most FCs are naturally cautious of challenging HCPs professional authority and intervening with the management plan of their patients. Active FCs involvement may lead some physicians to respond negatively to such FCs involvement.

In this study, some FC experienced full trust in HCPs knowledge and preferred to leave the decision to HCPs. These findings were in consistent with other studies such as Gainer et al., (2017), who found that decision making was centred on developing trust and faith in the surgeon rather than weighing different decision options.

Family Caregivers who have a health background are more able to ask, provide options and discuss with HCPs than those who lack the health background. Additionally, they enjoy a good professional relationship due to the common language and health experiences. One participant reported that she consulted other professionals whom she trust and changed the initial decision made by her husband Doctor. Thus, previous studies supported the current study findings and showed that effective relationship of HCPs with patients/family members is an important contributing factor of patient/family members involvement in D.M. (McCabeBennett et al., 2014; Vahdat et al., 2014)

\section{Roles of Family Caregivers in Decision-Making Process}

FCs play significant roles in triadic health D.M. Findings of the recent study revealed different roles of FC on D.M, concur with results of previous studies within different cultures(Doumit et al., 2008; R. Obeidat \& Khrais, 2016; Svedlund \& Danielson, 2004). It identified roles of advocator, informational support, and social support. All participants reported providing help to their patients and described this experience as a social support that represent Jordanian cultural expectations and obligations.

The most reported type of support provided by FCs was emotional support. Findings of this study have been previously reported by Boyle (2015);Al-Bahri et al (2017), in which FCs of a patient who don't have a healthcare background, their voice and presence meant a lot in the health encounters. Most of participants' experienced active roles in D.M process in line with other studies that reported family FCs provide informed support through seeking

Copyright (C) 2020, Journal of Scientific Research in Medical and Biological Sciences (JSRMBS), Under 
different resources to be able to participate actively in the D.M process (Gainer et al., 2017; R. F. Obeidat \& Lally, 2014).

Similar to previous studies results (Gainer et al., 2017; Lin et al., 2013), this study showed that FCs could be active advocators; they are able to defend patients benefits, give rich information, and ask questions that their patients may be unable to ask. Further, they act as translators for patients to better interact with HCPs and overcome language barriers. This role implies the nature of patient-family relationship. It was evident in this study that most FCs were in parental relationship that indicated a strong patient-family coalition. These findings is prevalent in Arabic Muslim culture. Strong parental relations among Jordanian caregivers is in agreement with Jordanian cultural imperative of family obligations towards ill individuals.

Involvement in D.M is significantly influenced by the values and culture of both patients/family and HCPs (AlHaqwi et al., 2015). Cultural background is the most important factor that influences the involvement of triadic D.M in health encounters. Individuals from western cultures perceive that patients' autonomy is above all factors. While those from Eastern culture as Jordan may not necessarily agree. Therefore, further understanding of the impact of different cultures on health care D.M is needed. On the other hand FCs who have a tense relation with their patients, particularly spouse relation, did not play active roles in the D.M process. Similarly, studies conducted by (Hirpara et al., 2016; Laidsaar-Powell et al., 2016; Lin et al., 2013) concluded that some FCs indirectly influence their patients choices in case patients formulated their own decisions.

The level of involvement in triadic encounters depends significantly on FCs characteristics and background. Family Caregivers as Health Care Providers play significant roles in D.M process; they provide full support and they are actively involved in health care decisions. Participants as RNs (F1 and F10) play advocate and collaborative roles to their patients as they have specialized health background. On the other hand, non-HCPs roles were limited to provide social and emotional support with least informational and collaborative roles.

The findings of this study are supported by previous studies(Al-Bahri et al., 2017; R. Obeidat \& Khrais, 2016; Yasein et al., 2017), in that FCs perceive the dominant role of HCPs in making decisions due to their specialized health knowledge. Family and patients unconditionally trusted their physicians and often accepted health management offered by them without asking any question. For instance, Al-Bahri et al (2017), can be explained that patients and their families lack specific health information to be actively involved in DM process. This might be attributed to the tight schedule of HCPs, mainly physicians, and being responsible to follow up the increased number of patients, which may reduce the chance to provide enough facts. In this study all participants are treated in public health sectors where large number of patients attended with limited HCPs numbers. However, this should not be a justification, since it is the patient/family rights to have the needed information about health condition(Yasein et al., 2017). 


\section{Conclusion and Suggestion}

Triadic decision-making among chronic cases is a complex process that involves patients, family and healthcare providers. They have different perceptions regarding patients' health needs. A transformation from HCPs centred approach to patient-family centred approach is needed as it was evident in most literature recommendations. Cultural background is the outmost factor influencing the final health decision-making. The researchers recommend considering family involvement, only in agreement with patients' preferences and wishes. Healthcare organizations and policy makers cannot make changes in isolation. The true engagement involves working in partnership with patients and families. (Carman et al., 2013)

There is an urgent need to find an ethically and socially acceptable balance between

the rights of the individual and familial influence in Asian societies, which is likely to remain a challenging task for many healthcare professionals in different health settings (Chong et al., 2015). Developing and establishing a systematic assessment of patients' and family members' needs and wishes in order to provide a specific-tailored support should become a priority for interdisciplinary clinical research in the near future.

\section{Conflict of Interest}

The authors of the article declare no conflict of interest.

\section{Funding:}

The authors received no funding for conducting this study.

\section{References}

Al-ananbeh, E., Abu-moghli, F., \& Khalaf, I. (2018). Perception and Experiences of Nurses Regarding Patients Involvement in Health Care Decisions : an Integrative Review. 7(2), 99-110.

Al-Bahri, A., Al-Moundhri, M., \& Al-Azri, M. (2017). The role of patients' families in cancer treatment decision-making: Perspectives among eastern and western families. Sultan Qaboos University Medical Journal, 17(4), e383-e385. https://doi.org/10.18295/squmj.2017.17.04.001

AlHaqwi, A. I., AlDrees, T. M., AlRumayyan, A., AlFarhan, A. I., Alotaibi, S. S., Alkhashan, H. I., \& Badri, M. (2015). Shared clinical decision making: A Saudi Arabian perspective. Saudi Medical Journal, 36(12), 1472-1476. https://doi.org/10.15537/smj.2015.12.13682

Alliance, F. C. (2015). Fact Sheet: Selected long-term care statistics. Family Caregiver Alliance: San Francisco, CA, USA.

Banning, M., Hafeez, H., Faisal, S., Hassan, M., \& Zafar, A. (2009). The impact of culture and sociological and psychological issues on Muslim patients with breast cancer in Pakistan. Cancer Nursing, 32(4), 317-324.

Barstow, C., Shahan, B., \& Roberts, M. (2018). Evaluating medical decision-making capacity 
in practice. American Family Physician, 98(1), 40-46.

Boyle, B. (2015). The critical role of family in patient experience. Patient Experience Journal, 2(2), 4-6. https://doi.org/10.35680/2372-0247.1112

Carman, K. L., Dardess, P., Maurer, M., Sofaer, S., Adams, K., Bechtel, C., \& Sweeney, J. (2013). Patient and family engagement: a framework for understanding the elements and developing interventions and policies. Health Affairs, 32(2), 223-231.

Charles, C., Gafni, A., \& Whelan, T. (1997). Shared decision-making in the medical encounter: What does it mean? (Or it takes, at least two to tango). Social Science and Medicine, 44(5), 681-692. https://doi.org/10.1016/S0277-9536(96)00221-3

Chong, J. A., Quah, Y. L., Yang, G. M., Menon, S., \& Krishna, L. K. R. (2015). Patient and family involvement in decision making for management of cancer patients at a centre in Singapore. BMJ Supportive and Palliative Care, 5(4), 420-426. https://doi.org/10.1136/bmjspcare-2012-000323

Dionne-Odom, J. N., Ejem, D., Wells, R., Barnato, A. E., Taylor, R. A., Rocque, G. B., Turkman, Y. E., Kenny, M., Ivankova, N. V, \& Bakitas, M. A. (2019). How family caregivers of persons with advanced cancer assist with upstream healthcare decisionmaking: A qualitative study. PloS One, 14(3), e0212967.

Doumit, M. A. A., Huijer, H. A. S., Kelley, J. H., \& Nassar, N. (2008). The lived experience of lebanese family caregivers of cancer patients. Cancer Nursing, 31(4). https://doi.org/10.1097/01.NCC.0000305751.28120.f4

Fishbein, M. (1975). Ajzen. i.(1975). Belief, Attitude, Intention and Behavior: An Introduction to Theory and Research.

Gainer, R. A., Curran, J., Buth, K. J., David, J. G., Légaré, J. F., \& Hirsch, G. M. (2017). Toward Optimal Decision Making among Vulnerable Patients Referred for Cardiac Surgery: A Qualitative Analysis of Patient and Provider Perspectives. Medical Decision Making, 37(5), 600-610. https://doi.org/10.1177/0272989X16675338

Hammami, M. M., Al Balkhi, A., De Padua, S. S., \& Abuhdeeb, K. (2020). Factors underlying surrogate medical decision-making in middle eastern and east Asian women: a Q-methodology study. BMC Palliative Care, 19(1), 137. https://doi.org/10.1186/s12904-020-00643-9

Hirpara, D. H., Cleghorn, M. C., Sockalingam, S., \& Quereshy, F. A. (2016). Understanding the complexities of shared decision-making in cancer: A qualitative study of the perspectives of patients undergoing colorectal surgery. Canadian Journal of Surgery, 59(3), 197-204. https://doi.org/10.1503/cjs.013415

Icek, A. (1991). The Theory of Planned Behavior Organizational Behavior and Human Decision Processes. Organizational Behavior and Human Decision Processes, 50(2), 179-211.

Javadi, M., Kadkhodaee, M., Yaghoubi, M., Maroufi, M., \& Shams, A. (2013). Applying Theory of Planned Behavior in Predicting of Patient Safety Behaviors of Nurses. Materia Socio Medica, 25(1), 52. https://doi.org/10.5455/msm.2013.25.52-55

Laidsaar-Powell, R., Butow, P., Bu, S., Charles, C., Gafni, A., Fisher, A., \& Juraskova, I. (2016). Family involvement in cancer treatment decision-making: A qualitative study of patient, family, and clinician attitudes and experiences. Patient Education and

Copyright (C) 2020, Journal of Scientific Research in Medical and Biological Sciences (JSRMBS), Under 
Counseling, 99(7), 1146-1155. https://doi.org/10.1016/j.pec.2016.01.014

Lamore, K., Montalescot, L., \& Untas, A. (2017). Treatment decision-making in chronic diseases: What are the family members' roles, needs and attitudes? A systematic review. Patient Education and Counseling, 100(12), 2172-2181.

https://doi.org/10.1016/j.pec.2017.08.003

Laryionava, K., Pfeil, T. A., Dietrich, M., Reiter-Theil, S., Hiddemann, W., \& Winkler, E. C. (2018). The second patient? Family members of cancer patients and their role in end-oflife decision making. BMC Palliative Care, 17(1), 1-9. https://doi.org/10.1186/s12904018-0288-2

Lin, M. L., Pang, M. C. S., \& Chen, C. H. (2013). Family as a whole: Elective surgery patients' perception of the meaning of family involvement in decision making. Journal of Clinical Nursing, 22(1-2), 271-278. https://doi.org/10.1111/j.13652702.2012.04194.x

McCabe-Bennett, H., Cassin, S. E., \& Antony, M. M. (2014). Naturalistic observation of perfectionistic behaviours. Unpublished Master's Thesis, Ryerson University, Toronto, Ontario, Canada.

Obeidat, R. F., Dickerson, S. S., Homish, G. G., Alqaissi, N. M., \& Lally, R. M. (2013). Controlling fear: Jordanian women's perceptions of the diagnosis and surgical treatment of early-stage breast cancer. Cancer Nursing, 36(6), 484-492. https://doi.org/10.1097/NCC.0b013e31826fc9b4

Obeidat, R. F., \& Lally, R. M. (2014). Health-related information exchange experiences of Jordanian women at breast cancer diagnosis. Journal of Cancer Education, 29(3), 548554. https://doi.org/10.1007/s13187-013-0574-X

Obeidat, R., \& Khrais, H. (2016). Jordanian physicians' attitudes toward disclosure of cancer information and patient participation in treatment decision-making. Asia-Pacific Journal of Oncology Nursing, 3(3), 281. https://doi.org/10.4103/2347-5625.189811

Polit, D. F., \& Beck, C. T. (2012). Nursing Research: Generating and Assessing Evidence for Nursing Practice. Lippincott Williams \& Wilkins.

Quinn, B. J. R., Schmitt, M., Baggs, J. G., Norton, S. A., Dombeck, M. T., \& Sellers, R. (2015). F @2012. 21(1), 43-52.

Sekimoto, M., Asai, A., Ohnishi, M., Nishigaki, E., Fukui, T., Shimbo, T., \& Imanaka, Y. (2004). Patients' preferences for involvement in treatment decision making in Japan. BMC Family Practice, 5, 1-10. https://doi.org/10.1186/1471-2296-5-1

Supportive, P. D. Q. (2019). Informal Caregivers in Cancer: Roles, Burden, and Support $(P D Q \circledR)$ : Health Professional Version.

Svedlund, M., \& Danielson, E. (2004). Myocardial infarction: Narrations by afflicted women and their partners of lived experiences in daily life following an acute myocardial infarction. Journal of Clinical Nursing, 13(4), 438-446. https://doi.org/10.1111/j.13652702.2004.00915.x

Teh, C. F., Karp, J. F., Kleinman, A., Reynolds, C. F., Weiner, D. K., \& Cleary, P. D. (2009). Older people's experiences of patient-centered treatment for chronic pain: A qualitative study. Pain Medicine, 10(3), 521-530. https://doi.org/10.1111/j.15264637.2008.00556.x 
Vahdat, S., Hamzehgardeshi, L., Hessam, S., \& Hamzehgardeshi, Z. (2014). Patient involvement in health care decision making: a review. Iranian Red Crescent Medical Journal, 16(1).

Yasein, N. A., Shakhatreh, F. M., Shroukh, W. A., S. Farah, M., \& Jaber, R. M. (2017). A Comparison between Patients' and Residents' Perceptions of Patient Centeredness and Communication Skills among Physicians Working at Jordan University Hospital. Open Journal of Nursing, 07(06), 698-706. https://doi.org/10.4236/ojn.2017.76052 